Comp Physiol. 2009;297(5):R1238-R1246.

12. Huo L, Maeng L, Bjorbaek C, Grill HJ. Leptin and the control of food intake: neurons in the nucleus of the solitary tract are activated by both gastric distension and leptin. Endocrinology. 2007;148(5):2189-2197.

13. Morton GJ, et al. Leptin action in the forebrain regulates the hindbrain response to satiety signals. J Clin Invest. 2005;115(3):703-710.

14. Ellacott KL, Halatchev IG, Cone RD. Characterization of leptin-responsive neurons in the caudal brainstem. Endocrinology. 2006;147(7):3190-3195.

15. Huo L, Grill HJ, Bjorbaek C. Divergent regulation of proopiomelanocortin neurons by leptin in the nucleus of the solitary tract and in the arcuate hypothalamic nucleus. Diabetes. 2006;55(3):567-573.

16. Mercer JG, Moar KM, Findlay PA, Hoggard N, Adam CL. Association of leptin receptor (OB-Rb), NPY and GLP-1 gene expression in the ovine and murine brainstem. Regul Pept. 1998;75-76:271-278.

17. Goldstone AP, et al. Leptin interacts with glucagon-like peptide-1 neurons to reduce food intake and body weight in rodents. FEBS Lett. 1997; 415(2):134-138.

18. Huo L, Gamber KM, Grill HJ, Bjorbaek C. Divergent leptin signaling in proglucagon neurons of the nucleus of the solitary tract in mice and rats.
Endocrinology. 2008;149(2):492-497.

19. Myers MG Jr, Leibel RL, Seeley RJ, Schwartz MW. Obesity and leptin resistance: distinguishing cause from effect. Trends Endocrinol Metab. 2010;21(11):643-651.

20. Munzberg H, Flier JS, Bjorbaek C. Region-specific leptin resistance within the hypothalamus of diet-induced obese mice. Endocrinology. 2004; 145(11):4880-4889

21. Ring LE, Zeltser LM. Disruption of hypothalamic leptin signaling in mice leads to early-onset obesity, but physiological adaptations in mature animals stabilize adiposity levels. J Clin Invest. 2010; 120(8):2931-2941.

\title{
Chronic lung allograft rejection and airway microvasculature: Is HIF-1 the missing link?
}

\author{
David S. Wilkes
}

Center for Immunobiology, Departments of Medicine, Microbiology and Immunology, Indiana University School of Medicine, Indianapolis, Indiana, USA.

\begin{abstract}
Chronic lung allograft rejection, known as obliterative bronchiolitis (OB), is the leading cause of death in lung transplant patients. Although $O B$ pathogenesis is not fully understood, in this issue of the JCI, Jiang and colleagues report that tissue hypoxia resulting in dysfunctional airway microvasculature precedes the airway fibrosis characteristic of $O B$. In addition, a relative deficiency of allograft endothelial cell-derived HIF- $1 \alpha$ contributes to this process. Data showing that overexpressing HIF-1 $\alpha$ restores the microvascular airway normoxia and prevents airway fibrosis highlight a novel role for vascular biology in $\mathrm{OB}$ pathogenesis.
\end{abstract}

Lung transplantation is the definitive therapy for many end-stage pulmonary diseases. However, the long-term survival of the lung transplant recipient is limited by chronic rejection known as obliterative bronchiolitis (OB, aka bronchiolitis obliterans syndrome [BOS]) (1). In fact, OB largely accounts for the 50\% five-year survival rate after lung transplantation, which is the worst of all solid organ allografts (1). The mechanisms leading to $\mathrm{OB}$ continue to be an area of intensive investigation, and studies have revealed that immune responses to the donor (alloimmunity) as well as exposure of autoantigens may participate in the rejection response (reviewed in ref. 2). The convergent result of these injurious activities is the development of a fibroproliferative process resulting in small airway occlusion, which is the pathologic hallmark of OB.

Conflict of interest: David S. Wilkes is the co-founder of ImmuneWorks Inc., which is developing novel forms of treatments for immunologically mediated lung diseases. The author has also received income as a consultant for ImmuneWorks Inc.

Citation for this article: J Clin Invest. 2011; 121(6):2155-2157. doi:10.1172/JCI58329.

\section{OB stems from hypoxia}

Implicit in the term chronic rejection is the idea that immune mechanisms are likely to predominate in $\mathrm{OB}$. However, even prior to immune activation, the lung may be at risk for OB due to airway hypoxia resulting from transplant-related microvasculature interruption. The lung has three vascular supplies, the pulmonary arteries, the pulmonary veins, and the bronchial artery, and is the only solid organ for which allograft does not involve direct systemic arterial reconnection at the time of surgery. The pulmonary arterial/venous circulation is restored in the transplanted lung. However, the bronchial artery, the only source of fully oxygenated blood under systemic arterial pressure, is not reanastomosed after transplantation due to the significant technical complexities associated with this procedure.

The lack of an intact bronchial artery circulation leads to impaired microcirculation, suggesting that prolonged airway hypoxia contributes to $O B$. In fact, previous studies from the Nicolls group have confirmed that airway epithelial hypoxia occurs following clinical lung transplantation (3), and other researchers have reported that the loss of the microvasculature in small airways precedes OB $(4,5)$. Hypoxia, a key adverse effect of losing the vascular supply, may induce profound changes in airway epithelium. One of these effects could be the induction of epithelial mesenchymal transition (EMT), a process implicated in fibrogenesis in many organs, including the lung (6). Indeed, studies from the laboratory of Jacob Sznajder demonstrated that both moderate and severe hypoxia induced EMT (6). These findings have direct relevance to lung transplantation, since recent studies have detected EMT in OB lesions (7-9).

Recent studies strongly suggest that hypoxia may lower the threshold to induce adaptive immune responses known to have key roles in acute lung transplant rejection. Due to the presence of bronchus-associated lymphoid tissue, interstitial and interepithelial dendritic cells, a full complement of lymphocytes, and macrophages, the lung is uniquely able to mount adaptive immune responses in the absence of any secondary lymphoid organs $(10,11)$. Indeed, in essence, the lung is a lymph node with alveoli (2). What is the relationship of immunity to chronic hypoxia and rejection in the transplanted lung? Recent studies indicate that hypoxia may augment immune activation (12) and that alloimmune activation occurs within the transplanted lung (10). For example, hypoxia induces the activation of dendritic cells that stimulate alloimmunity, produce proinflammatory cytokines, and activate Th17 cells that produce IL-17 $(13,14)$. In addition, production of IL-17 
is strongly correlated with OB (15). Collectively, these studies suggest that hypoxia may lead to augmented allo- and autoimmunity injury that further predisposes to fibrogenesis. It is well documented that calcineurin inhibitors (CNI), the mainstay of posttransplant immunosuppressive therapy, may also be fibrogenic. Therefore, delivery of these agents, either systemically or via the inhaled route, is likely not to prevent $\mathrm{OB}$, but instead could actually contribute to fibrogenesis in part due to airway hypoxia that results from a lack of an intact and robust airway microvasculature. Indeed, widespread CNI use could help to explain why $75 \%$ of lung transplant recipients develop OB (1).

\section{A new direction for prevention?}

If the loss of microvasculature after lung transplantation results in hypoxia leading to airway fibrosis, then normoxia via microvascular repair should prevent fibrosis. Indeed, data derived from a unique preclinical model reported by Jiang et al. in the current issue of the JCI fully support this hypothesis (16). This work is an extension of a prior study from the same group and reported previously in the JCI (17). Utilizing a mouse model of orthotopic airway allograft transplantation, the researchers found that restoration of airway microvasculature via local overexpression of HIF- $1 \alpha$ not only resulted in normoxic conditions, but also prevented airway fibrosis. Moreover, the authors show that endogenous HIF-1 $\alpha$ expression was limited to donor, and not recipient, endothelial cells (16). Although constitutive HIF-1 $\alpha$ expression occurred following airway transplantation, it was not sufficient to prevent the fibrotic response. Notably, vascular bed development was HIF-1 $\alpha$ dependent, since revascularization was profoundly limited in allografts genetically deficient in HIF- $1 \alpha$. In addition, the rate of chronic rejection was accelerated markedly in HIF- $1 \alpha-$ deficient and wild-type grafts, whereas overexpressing HIF- $1 \alpha$ prevented fibrosis and delayed the onset of OB. These data are consistent with a study from Belperio et al., who reported angiogenesis occurring after loss of the microvasculature actually facilitated fibrosis in models of obliterative airway disease (18). Collectively, these studies demonstrate that not only is hypoxia a major risk factor for fibrosis that characterizes chronic rejection, but also that a dysfunctional vascular bed with associated hypoxia exacerbates the fibrotic response.

\section{Why HIF-1?}

There are many proangiogenic mediators, so one must question the reasoning behind a specific focus on HIF-1. The induction of virtually all known angiogenic growth factors is HIF-1 $\alpha$ dependent, and hypoxia is a potent stimulator for HIF- $1 \alpha$ (reviewed in ref. 19). There are many clinical implications of the current study. Immune modulation after transplantation has been and continues to be the primary focus of strategies to prevent $\mathrm{OB}$; however, there are no effective immunosuppressive therapies for chronic rejection. The study from Jiang et al. provides hope in this regard. HIF- $1 \alpha$-induced revascularization slowed the rate of chronic rejection despite the presence of alloreactive $\mathrm{T}$ cells that are known to mediate the rejection response. Furthermore, this protective effect was augmented when the immune system was suppressed via costimulatory blockade. These data also suggest that hypoxia induced by a dysregulated vascular supply likely predisposes the airway to alloimmune-induced injury (16). Accordingly, restoration of a robust airway microvasculature in the presence of appropriate immunosuppression might prevent chronic rejection. In other words, it is interesting to speculate that the inability to treat $\mathrm{OB}$ with currently utilized immunosuppressive regimens could be in part due to airway hypoxia that results from an aberrant airway microvasculature.

Data from the current report strongly suggest that airway microvasculature preservation should be a desired outcome immediately after transplantation and during acute rejection episodes when the microvascular bed is under immune attack. Unfortunately, preservation of the airway microvasculature by donor-recipient bronchial artery anastomosis at the time of transplantation may not prevent $\mathrm{OB}$, since acute rejection-induced microvascular loss could occur virtually any time after transplant. It is easy to surmise from the data presented by Jiang et al. that HIF- $1 \alpha$ could be a potential therapeutic in clinical lung transplantation to preserve the microvasculature, thereby preventing tissue hypoxia/fibrosis. Pharmacologic agents that could be used in this regard do exist; desferoxamine and $\mathrm{CoCl}_{2}$ have been reported to induce HIF-1 expression in animal models of ischemic injury (19). However, toxicities and nonspecific actions would preclude the clinical use of such agents in lung transplantation. Similar to the current study, HIF-1 $\alpha$ gene therapy has been efficacious in restoring vascular integrity in other preclinical models (20), but the potential limitations of adenoviralmediated gene therapy in the clinical setting have been well documented.

\section{Future directions}

What is the future of determining the effects of airway microvasculature preservation and repair in the transplanted lung on clinical OB? I envision a two-step process. The first step will be to conduct randomized trials of bronchial artery anastomosis at the time of lung transplantation followed by an assessment of OB. If bronchial anastomosis fails to prevent the development of $\mathrm{OB}$, it is possible that acute rejection-induced microvasculature loss is inevitable after transplantation. If that is the case, the second step will be to develop strategies to deliver HIF- $1 \alpha$ to sites of active acute rejection in the presence of augmented immunosuppression to determine whether OB may be prevented. Robust animal models will facilitate the development of new therapeutics. Although a mouse model of orthotopic lung transplantation that develops $\mathrm{OB}$ has been developed recently (21), technical limitations preclude the use of this model to study bronchial artery reanastomosis. However, such a model could be the platform for studying novel approaches for delivery of HIF- $1 \alpha$ to the transplanted lung. Finally, studies of OB have focused almost exclusively on various aspects of immune regulation. The current report from Jiang et al. identifies a role for vascular biology in chronic rejection and is a major step forward in understanding the nonimmune mechanisms at play in the pathogenesis of this lethal disease. Accordingly, this report is also a clarion call for continued in-depth investigations into the nonimmune pathways that contribute to OB to identify novel targets for therapeutic intervention.

\section{Acknowledgments}

This work is supported by NIH grants RO1HL60797, RO1096845, and PO1AI084853 to D.S. Wilkes.

Address correspondence to: David S. Wilkes, Indiana University School of Medicine, 340 West 10th Street, Suite 6200, Indianapolis, Indiana, 46202, USA. Phone: 317.278.7020; Fax 317.274.8439; E-mail: dwilkes@iupui.edu. 
1. Studer SM, Levy RD, McNeil K, Orens JB. Lung transplant outcomes: a review of survival, graft function, physiology, health-related quality of life and cost-effectiveness. Eur Respir J. 2004;24(4):674-685.

2. Shilling RA, Wilkes DS. Immunobiology of chronic lung allograft dysfunction: new insights from the bench and beyond. Am J Transplant. 2009; 9(8):1714-1718.

3. Dhillon GS, et al. Lung transplant airway hypoxia: a diathesis to fibrosis? Am J Respir Crit Care Med. 2010;182(2):230-236.

4. Luckraz H, et al. Microvascular changes in small airways predispose to obliterative bronchiolitis after lung transplantation. J Heart Lung Transplant. 2004;23(5):527-531.

5. Luckraz H, Goddard M, McNeil K, Atkinson C, Sharples LD, Wallwork J. Is obliterative bronchiolitis in lung transplantation associated with microvascular damage to small airways? Ann Thorac Surg. 2006;82(4):1212-1218.

6. Zhou G, et al. Hypoxia-induced alveolar epithelialmesenchymal transition requires mitochondrial ROS and hypoxia-inducible factor 1. Am J Physiol Lung Cell Mol Physiol. 2009;297(6):L1120-L1130.

7. Willis BC, Borok Z. Epithelial-mesenchymal tran- sition: potential role in obliterative bronchiolitis? Thorax. 2009;64(9):742-743.

8. Borthwick LA, et al. Epithelial to mesenchymal transition (EMT) and airway remodelling after human lung transplantation. Thorax. 2009;64(9):770-777.

9. Ward C, et al. Phenotype of airway epithelial cells suggests epithelial to mesenchymal cell transition in clinically stable lung transplant recipients. Tho$\operatorname{rax} .2005 ; 60(10): 865-871$.

10. Gelman AE, et al. Cutting edge: Acute lung allograft rejection is independent of secondary lymphoid organs. J Immunol. 2009;182(7):3969-3973.

11. Constant SL, et al. Resident lung antigen-presenting cells have the capacity to promote Th2 T cell differentiation in situ. JClin Invest. 2002;110(10):1441-1448.

12. Kamler M, et al. Bronchial artery revascularization restores peribronchial tissue oxygenation after lung transplantation. J Heart Lung Transplant. 2004;23(6):763-766.

13. Wang $Q$, et al. Reoxygenation of hypoxia-differentiated dentritic cells induces Th1 and Th17 cell differentiation. Mol Immunol. 2010;47(4):922-931.

14. Jantsch J, et al. Hypoxia and hypoxia-inducible factor- 1 alpha modulate lipopolysaccharide-induced dendritic cell activation and function. J Immunol.
2008;180(7):4697-4705

15. Burlingham WJ, et al. IL-17-dependent cellular immunity to collagen type $\mathrm{V}$ predisposes to obliterative bronchiolitis in human lung transplants. J Clin Invest. 2007;117(11):3498-3506.

16. Jiang X, et al. Adenovirus-mediated HIF- $1 \alpha$ gene transfer promotes repair of mouse airway allograft microvasculature and attenuates chronic rejection. J Clin Invest. 2011;121(6):2336-2349.

17. Babu AN, et al. Microvascular destruction identifies murine allografts that cannot be rescued from airway fibrosis. J Clin Invest. 2007;117(12):3774-3785.

18. Belperio JA, et al. Critical role for CXCR3 chemokine biology in the pathogenesis of bronchiolitis obliterans syndrome. JImmunol. 2002;169(2):1037-1049.

19. Rey S, Semenza GL. Hypoxia-inducible factor-1dependent mechanisms of vascularization and vascular remodelling. Cardiovasc Res. 2010;86(2):236-242.

20. Bosch-Marce M, et al. Effects of aging and hypoxiainducible factor- 1 activity on angiogenic cell mobilization and recovery of perfusion after limb ischemia. Circ Res. 2007;101(12):1310-1318.

21. Fan L, et al. Neutralizing IL-17 prevents obliterative bronchiolitis in murine orthotopic lung transplantation. Am J Transplant. 2011;11(5):911-922.

\section{The yin, the yang, and the Angiopoietin-1}

\section{Pipsa Saharinen and Kari Alitalo}

Molecular/Cancer Biology Program and Finnish Institute for Molecular Medicine, University of Helsinki, Helsinki, Finland.

\begin{abstract}
Twenty years after the discovery of the vascular endothelial Tie receptor tyrosine kinases and 15 years after the discovery of the Tie2 ligand, angiopoietin-1 (Angpt1, also known as Ang1), a study published in the current issue of the JCI reveals an unexpected loss-of-function phenotype of mice conditionally deleted of the Angpt1 gene. The results suggest that Angpt1 is needed as a vascular stabilizing factor that organizes and limits the angiogenesis response and protects from pathological consequences, such as tissue fibrosis.
\end{abstract}

\section{Angiopoietin-1 and angiopoietin-2 in sprouting angiogenesis}

In the process of sprouting angiogenesis that typically occurs when VEGF stimulates capillaries, the leading edges of growing capillary sprouts display migrating and slowly dividing tip cells, which extend filopodia in response to VEGF gradients (1). Tip cells are followed by less mobile proliferating stalk cells, which express the Tie2 receptor and recruit pericytes. Angiopoietin-1 (Angpt1) is expressed by perivascular cells, such as pericytes, and the related Tie2 ligand Angpt2 is expressed by the endothelial tip cells. Although Angpt 1 and Angpt 2 both bind to Tie2 with similar affinities, they seem to act as antagonists of each other in several vascular processes

Conflict of interest: The authors declare research grant support from Medimmune, LLC.

Citation for this article: J Clin Invest. 2011; 121(6):2157-2159. doi:10.1172/JCI58196.
$(2,3)$. In the stalk cells that become associated with pericytes, Angpt 1 may limit angiogenesis by inducing homomeric Tie2 complexes across the cell-cell junctions, and mediating cell-cell adhesion, antipermeability, and cell survival $(4,5)$. In contrast, Angpt2 may regulate cell-matrix interactions in the growing vessels to facilitate sprouting (Figure 1).

\section{Myocardial Angpt1 is essential for cardiovascular development}

The study by Jeansson et al. shows that despite the constitutive expression of Angpt 1 in many cell types, including perivascular mural cells, and constitutive Tie2 activation in vascular endothelial cells in vivo $(2,6)$, Angpt 1 is not necessary for normal steady-state physiological processes in the adult, being dispensable in the blood vasculature from E13.5 onward (7). However, Angpt 1 is essential for the development of embryonic vasculature during a short period between E10.5 and E13.5 $(7,8)$. Tie 2 and the related Tie1 (9) are also critical during that period. Analysis of Tie2-deleted and mosaic mutant embryos at E10.5 indicated that Tie 2 is required in the developing endocardium for myocardial attachment and trabeculation, whereas Tie 2 and Tie 1 are dispensable for the initial assembly of the rest of the vasculature (10-12). Thus, it was of no surprise that the embryos died when, in the study by Jeansson et al., Angpt1 was deleted from the developing embryos before E12.5 (7). Interestingly, however, these new data indicate that cardiomyocyte-specific deletion of Angpt1 reproduces much of the phenotype of the full Angpt1 knockout, suggesting that hemodynamic problems propagate the vascular defects to other tissues $(7,8)$. However, studies in which Angpt 1 would be deleted in other developing vascular beads, while leaving the cardiac Angpt1 levels intact, are needed to confirm these results. The previously published mosaic analysis indicated that both Tie 1 and Tie 2 are required in the microvasculature during late organogenesis and in essentially all blood vessels of the adult (12, 13). The report by Jeansson et al. thus raises the intriguing possibility that the requirement for Tie 1 and Tie2 in the microvasculature is independent of Angpt1 function. 